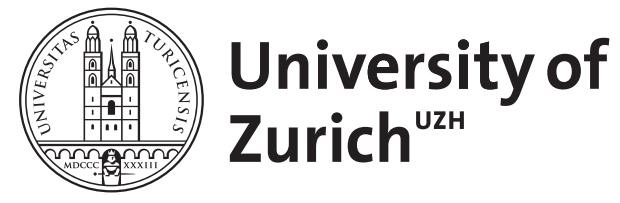

\title{
Über Wiederholungen, Murmeltiere und Kreditkarten
}

Wolff, Eberhard

\begin{abstract}
Ausgangspunkt dieser Gedanken ist der Film «Und täglich grüsst das Murmeltier». In ihm erlebt ein fieser Wettermoderator einen schlimmen Tag bei schlechtem Wetter immer und immer wieder. Es geht also um Wiederholungen, die es ja auch in der Medizin gibt - etwas bei Operationen.
\end{abstract}

Posted at the Zurich Open Repository and Archive, University of Zurich ZORA URL: https://doi.org/10.5167/uzh-92275

Journal Article

Published Version

Originally published at:

Wolff, Eberhard (2013). Über Wiederholungen, Murmeltiere und Kreditkarten. Schweizerische Aerztezeitung, 94(25):1008. 


\section{Über Wiederholungen, Murmeltiere und Kreditkarten}

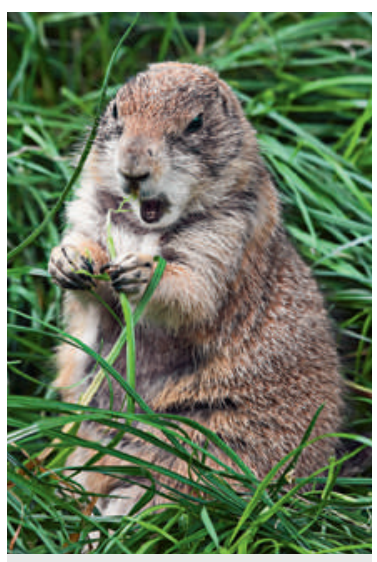

Ein «Groundhog», der amerikanische Kollege des Alpenmurmeltiers, das zumindest im Film täglich grüsst.
Heute einmal etwas ganz Unmedizinisches. Kennen Sie den zwanzig Jahre alten, immer noch wunderbaren Film «Und täglich grüsst das Murmeltier» (englisch «Groundhog Day») mit Bill Murray? Darin erliegt der fiese Wettermoderator Phil Connors einem Zeitschleifen-Fluch und muss einen schlimmen Tag in der ungeliebten Provinz bei schlechtem Wetter immer und immer wieder neu durchleben. Wie ich darauf komme? Dazu später.

Kürzlich erhielt ich eine Werbebroschüre einer Kreditkartenfirma. Mit ihrem Produkt könne ich in einem Jahr Rabattpunkte in sechsstelliger Zahl erhalten, wenn ich nur überall die Karte einsetze. Ein Beispieltag: Morgens Zeitung und Kaffee kaufen. Dann: Uups! Wegen eines Flecks ein neues Hemd besorgen müssen. Zum Lunch wird die Freundin eingeladen. Nach der Arbeit einen Blumenstrauss und Lebensmittel einkaufen, tanken und am Abend noch ein Buch im Internet bestellen. Summa summarum etwa 370 Franken umgesetzt. Wunderbar: Mit den so gewonnenen Rabattpunkten kann man nach 365 Tagen ist, alle Blumen, Bücher und Hemden wieder wegzuschaffen.

Mit den Wiederholungen ist es so eine Sache. Sie werden schnell unangenehm, weil wir die Abwechslung lieben. Für Phil Connors ist die Wiederholung ein Fluch. Er kommt nicht aus der Zeitschleife heraus und muss in seiner widrigen Lage immer wieder neu anfangen. Und manch einem von Ihnen mag das ebenso gehen: tagein, tagaus Knie-Operationen durchführen, weil man die eben so gut kann. Da wird die Routine zur Monotonie.

Aber warum sind vom Tatort mittlerweile 877 Folgen gedreht worden, und er ist immer noch erfolgreich? Warum sind Serien so ungemein im Trend? Spätestens mit Andy Warhol haben wir gesehen, dass die Serialität ein Beitrag zur Ästhetik der Moderne ist [1]. In der Routine der Wiederholung steckt nämlich nicht nur Erwartungssicherheit (dass der Mörder gefasst wird), sondern auch eine Chance auf einen Ausbruch aus der Routine! Es kommt einfach darauf an, was man aus den Wiederholungen macht.

\section{«Es kommt einfach darauf an, was man aus den Wiederholungen macht.»}

* PD Dr. rer. soc. Eberhard Wolff ist Kulturwissenschaftler, Medizinhistoriker und Mitglied der Redaktion Medizingeschichte der Schweizerischen Ärztezeitung.

eberhard.wolff[at]saez.ch einen Freiflug nach Hawaii und zurück ergattern. Ach ja: Das erfordert allein für Essen, Benzin und Hemden usw. 135000 Franken Jahresumsatz. Ich habe den Kartenantrag dann erst einmal beiseite gelegt.

Stellen Sie sich das PR-Szenario auch jenseits der Kosten einmal ganz konkret vor: Zeitung, Kaffee, Lebensmittel: Klar, braucht man täglich. Auch am Sonntag? Hmmm. Lunch mit der Freundin? OK, sogar sieben $\mathrm{Mal}$ in der Woche, wenn die Liebe gross ist. Wenn die Liebe sehr gross ist, will man die Angebetete sogar jedes Mal einladen.

Danach wird es schwieriger. Blumen verwelken schnell, aber jeden Tag einen Blumenstrauss, das schafft auf die Dauer Platzprobleme. Jeden Tag ein Buch, das bedeutet mit der Zeit gewisse Herausforderungen an die Lese- und Lagerungskapazitäten. Richtig grausam wird es aber erst, wenn man jeden Tag einen Fleck aufs Hemd machen muss. Das ist ungefähr so wie Bill Murray alias Phil Connors, der in seiner Zeitschleife jeden Tag in die gleiche Riesenpfütze tritt und sich masslos darüber ärgert. Beruhigend ist bei unserem Kreditkarten-Szenario allenfalls, dass das tägliche Volltanken schon alleine dafür nötig
Phil Connors befreit sich aus dem ZeitschleifenFluch, indem er vom Misanthrop und Zyniker zum guten Menschen mutiert. Er entwickelt wahre Liebe und gewinnt die angebetete Produzentin Rita (alias Andie MacDowell). Mit Liebe allein schafft er es aber nicht. Erst die Routine unendlich vieler Anläufe ermöglicht es ihm, sie kennenzulernen und ihr Herz zu erobern - und nebenbei auch die Riesenpfütze zu umgehen.

Ähnlich ist es mit der Kreditkarte (und vielleicht auch mit der Knie-Operation): Erst die ständige Wiederholung ihres Zückens bringt einen aus der Monotonie heraus nach Hawaii oder anderswo hin. Vielleicht sollte ich mir das mit dem Kreditkartenantrag doch noch einmal durch den Kopf gehen lassen.

Eberhard Wolff*

1 Kelleter F. (Hrsg.) Populäre Serialität: Narration-Evolution-Distinktion. Zum seriellen Erzählen seit dem 19. Jahrhundert. Bielefeld: transcript; 2012. 